\title{
SEQUENCES OF REALS \\ TO SEQUENCES OF ZEROS AND ONES
}

\author{
STEVEN KALIKOW
}

(Communicated by Andreas R. Blass)

\begin{abstract}
This paper considers maps from sequences of reals to sequences of zeros and ones which preserve the tail. The continuum hypothesis is used.
\end{abstract}

\section{INTRODUCTION}

In this paper $\mathbf{N}$ does not include 0 .

Let $A=\mathscr{R}^{\mathbf{N}}$ and $B=\{0,1\}^{\mathbf{N}}$. The purpose of this paper is to discuss certain functions $F: A \rightarrow B$. A function $F: A \rightarrow B$ is said to be good if:

(1) for $\left\{a_{i}\right\} \in A,\left\{b_{i}\right\} \in A$, (there exists $N$ such that $n>N \rightarrow a_{n}=b_{n}$ ) iff there exists $M$ such that $m>M \rightarrow\left(F\left(\left\{a_{i}\right\}\right)_{m}=\left(F\left(\left\{b_{i}\right\}\right)\right)_{m}\right.$;

(2) $\left(F\left(\left\{a_{i}\right\}\right)\right)_{m}$ depends only on $a_{1}, a_{2}, \ldots, a_{m}$.

A function $F: A \rightarrow B$ is said to be $m$-good if:

(a) condition 1 above;

(b) $F$ is measurable (Lebesgue measure and $\sigma$-algebras).

The purpose of this paper is to show the continuum hypothesis implies the existence of good maps (see the note below); and $m$-good maps do not exist. The first result is much deeper than the second.

Question: Does the converse of 1 hold?

Note. We prove (1) by proving the existence of a good map from $\aleph_{1}^{\mathbf{N}} \rightarrow$ $\{0,1\}^{\mathbf{N}}$ (we do not need continuum hypothesis for this). Then, we get (1) by assuming $\aleph_{1}=C$.

\section{1. $m$-GOOD MAPS DO NOT EXIST}

Elements of $\mathscr{R}^{\mathbf{N}}$ can be considered as sequences of reals, where $r_{n}$ is the $n$th real, and each $r_{n}$ can be considered to be a sequence of zeros and ones where $r_{n, j}$ is the $j$ th entry of $r_{n}$. In this manner, elements of $\mathscr{R}^{\mathbf{N}}$ can be regarded as two-dimensional sequences $r_{n, j}, n, j \in \mathbf{N}$, of zeros and ones. Let

Received by the editors June 27, 1988; revised March 10, 1989.

1980 Mathematics Subject Classification (1985 Revision). Primary 04A30, 03E05. 
$u$ be $\frac{1}{2}, \frac{1}{2}$ product measure on all these zeros and ones. I will write $P$ instead of $u$, connoting probability of.

Now fix $n_{0}, j_{0}$ and suppose we simply alter $r_{n_{0}, j_{0}}$ to $\hat{r}_{n_{0}, j_{0}}=1-r_{n_{0}, j_{0}}$ keeping all other $r_{n, j}$ fixed. The output $\left\{b_{i}\right\}$ in $\{0,1\}^{N}$ will alter to $\left\{\hat{b}_{i}\right\}$, $i \in \mathbf{N}$.

Now change all $r_{n_{0}, j}$ to zero and thereby alter all $\left\{b_{i}\right\}$ to $\hat{b}_{i}$. By property 1 there exists $g\left(n_{0}\right)$ such that

$$
P\left(\text { for all } i>g\left(n_{0}\right), \quad b_{i}=\hat{\hat{b}}_{i}\right)>1-\frac{1}{2 \cdot(100)^{n_{0}}} .
$$

By symmetry

$$
P\left(\text { for all } i>g\left(n_{0}\right), \quad \hat{b}_{i}=\hat{b}_{i}\right)>1-\frac{1}{2 \cdot(100)^{n_{0}}} .
$$

Therefore

$$
P\left(\text { for all } i>g\left(n_{0}\right), \quad b_{i}=\hat{b}_{i}\right)>1-\left(\frac{1}{100}\right)^{n_{0}} .
$$

Note that $g\left(n_{0}\right)$ depends only on $n_{0}$ and not on $j_{0}$.

By property 2 , for every $n_{0}$ there exists $n_{1}$ such that $b_{1}, b_{2}, \ldots, b_{g\left(n_{0}\right)}$ will all be practically determined by $\left\{r_{i, j}: i \leq n_{1}\right.$, and $\left.j \leq n_{1}\right\}$. Thus fixing $n_{0}$, choosing $n_{1}$ sufficiently large, choosing $j_{0}>n_{1}$, and as before, switching $r_{n_{0}, j_{0}}$ to $r_{n_{0}, j_{0}}=1-r_{n_{0}, j_{0}}$ we get

(4) $P\left(\right.$ for $\left.i \in\left\{1,2, \ldots, g\left(n_{0}\right)\right\}, \quad b_{i}=\hat{b}_{i}\right)>1-\left(\frac{1}{100}\right)^{n_{0}}$.

3 and 4 yield

(5) $P$ (for every $n_{0}$, there exists $j_{0}$, such that when $r_{n_{0}, j_{0}}$ is altered to $1-r_{n_{0}, j_{0}}$ the output is not altered) $>1-2\left(\frac{1}{100}\right)^{n_{0}}$.

Summing up over $n_{0}$ we get that there is positive probability after altering all $r_{n_{0}}$, that the output will remain unchanged. This contradicts condition 1 .

\section{Good MAPS EXIST}

A map from one collection of sequences to another collection of sequences is called "good" if it obeys the conditions of "good" in the Introduction. Clearly, the property "good" is closed under composition.

Let $\mathbf{R}^{\mathbf{N}}$ be sequences of reals.

Let $\aleph_{1}^{N}$ be sequences of ordinals less than $\aleph_{1}$.

Let $\widehat{\aleph_{1}^{N}}$ be sequences of ordinals less than $\aleph_{1}$ without repetition.

Let $L$ be sequences of finite sequences of countable ordinals $\left\{L_{i}\right\}_{i=1}^{\infty}$ such that all but finitely many of the countable ordinals which occur in $\bigcup_{i=1}^{\infty} L_{i}$ occur in infinitely many of the $L_{i}$.

Let $\mathbf{N}^{\mathbf{N}}$ be sequences of non-negative integers.

Let $\{0,1\}^{N}$ be sequences of zeros and ones. 
We will show that there is a good map from $\mathbf{R}^{\mathbf{N}}$ to $\{0,1\}^{\mathbf{N}}$ by showing that there is a good map from $\mathbf{R}^{\mathbf{N}}$ to $\aleph_{1}^{\mathbf{N}}$, from $\aleph_{1}^{\mathbf{N}}$ to $\widehat{\aleph_{1}^{\mathbf{N}}}$, from $\widehat{\aleph_{1}^{\mathbf{N}}}$ to $L$, from $L$ to $\mathbf{N}^{\mathbf{N}}$, and from $\mathbf{N}^{\mathbf{N}}$ to $\{0,1\}^{\mathbf{N}}$ :

(a) From $\mathbf{R}^{\mathbf{N}}$ to $\aleph_{1}^{\mathbf{N}}$. Using the continuum hypothesis $\mathbf{R}^{\mathbf{N}}$ and $\aleph_{1}^{\mathbf{N}}$ can be regarded as identical. This is the only use of the continuum hypothesis in this paper.

(b) From $\aleph_{1}^{\mathbf{N}}$ to $\widehat{\aleph_{1}^{\mathbf{N}}}$. Let $\Phi$ be a bijection from $\aleph_{1} \times \mathbf{N}$ to $\aleph_{1}$; the map $\varnothing: \aleph_{n}^{\mathbf{N}} \rightarrow \widehat{\aleph_{1}^{\mathbf{N}}}$ defined by $\left(a_{1}, a_{2}, \ldots,\right) \mapsto\left(\Phi\left(a_{1}, 1\right), \Phi\left(a_{2}, 2\right) \ldots\right)$ is good.

(c) From $\widehat{\aleph_{1}^{\mathrm{N}}}$ to $L$.

The following proof is due to the referee. It is simpler than my original proof.

For each ordinal $\alpha<\aleph_{1}$, let $D_{n}^{\alpha}$ be a nondecreasing sequence of finite sets whose union is $\alpha$. Given any sequence $\left\{\alpha_{n}\right\}_{n=1}^{\infty}$, then $\varnothing\left(\left\{\alpha_{n}\right\}_{n=1}^{\infty}\right)=\left\{F_{n}\right\}_{n=1}^{\infty}$ where $F_{n}$ is a list in increasing order of the smallest set of ordinals $F$, such that $\left\{\alpha_{m}: m \leq n, \alpha_{m} \leq \alpha_{n}\right\} \subseteq F$ and for any $B \in F, D_{n}^{B} \subseteq F$.

We now prove $\varnothing$ to be a good map. This means that if $\varnothing\left(\left\{\alpha_{n}\right\}_{n=1}^{\infty}\right)=\left\{F_{n}\right\}_{n=1}^{\infty}$ and $\varnothing\left(\left\{\beta_{n}\right\}_{n=1}^{\infty}\right)=\left\{G_{n}\right\}_{n=1}^{\infty}$ then

(i) If $\alpha_{n} \neq \beta_{n}$ for infinitely many $n$, then $F_{n} \neq G_{n}$ for infinitely many $n$.

(ii) If $\alpha_{n} \neq \beta_{n}$ for only finitely many $n$, then $F_{n} \neq G_{n}$ for only finitely many $n$.

(iii) $F_{n}$ depends only on $\alpha_{1}, \alpha_{2}, \ldots, \alpha_{n}$.

Proof of (i). For a fixed $n$, suppose $\alpha_{n} \neq \beta_{n}$. Suppose W.L.O.G. $\alpha_{n}>\beta_{n}$. Then $\alpha_{n} \in F_{n}$ but $\alpha_{n} \notin G_{n}$ so $F_{n} \neq G_{n}$.

Proof of (ii). There exists $N_{1}$ such that $\alpha_{n}=\beta_{n}$ for all $n \geq N_{1}$. Because there is no repetition on $\left\{\alpha_{n}\right\}$ or $\left\{\beta_{n}\right\}$, there exists $N_{2}$ so that for all $n \geq N_{2}$,

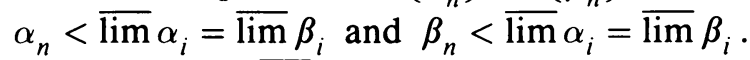

For any $\gamma<\overline{\lim } \alpha_{i}$, let $f(\gamma)$ be the least member of the set

$$
\left\{\alpha_{i}: i>\max \left(N_{1}, N_{2}\right) \text { and } \alpha_{i}>\gamma\right\} \text {. }
$$

For each $\gamma<\varlimsup \lim \alpha_{i}$ let $g(\gamma)=n$, where $n$ is chosen so that $\left\{\alpha_{i}: i \leq\right.$ $\left.\max \left(N_{1}, N_{2}\right) \wedge \alpha_{i}<\gamma\right\} \cup\left\{\beta_{i}: i \leq \max \left(N_{1}, N_{2}\right) \wedge \beta_{i}<\gamma\right\} \subset D_{n}^{\gamma}$. I claim that $F_{n}=G_{n}$ for all $n>$

$$
\begin{aligned}
\max & \left(\left\{m: \exists i, \alpha_{m}=f\left(\alpha_{i}\right) \wedge i \leq \max \left(N_{1}, N_{2}\right) \wedge \alpha_{i}<\varlimsup\right.\right. \\
& \cup\left\{m: \exists i, \beta_{m}=f\left(\beta_{i}\right) \wedge i \leq \max \left(N_{1}, N_{2}\right) \wedge \beta_{i}<\varlimsup\right. \\
& \cup\left\{g\left(f\left(\alpha_{i}\right)\right): i<\max \left(N_{1}, N_{2}\right), \alpha_{i}<\varlimsup\right. \\
& \cup\left\{g\left(f\left(\beta_{i}\right)\right): i<\max \alpha_{j}\right\} \\
&
\end{aligned}
$$

Fix $n$ as above. Let $S=\left\{a_{m}: m \leq n\right.$ and $\left.a_{m} \leq a_{n}\right\}$. We are given that $S \subset F_{n}$. The reader can verify that to prove $F_{n} \subset G_{n}$ it suffices to prove that 
$S \subset G_{n}$. We prove $G_{n} \subset F_{n}$ similarly. It is trivial by the definition of $N_{1}$ that $S \cap\left\{\alpha_{m}: m \geq N_{1}\right\} \subset G_{n}$. We need only prove that $S \cap\left\{\alpha_{m}: m \leq N_{1}\right\} \subset G_{n}$. Since $S \cap\left\{a_{m}: m<N_{1}\right\}$ is finite, choose the largest element $\alpha_{j}, f\left(\alpha_{j}\right) \in$ $S \cap\left\{\alpha_{m}: m \geq N_{1}\right\}$ so $f\left(\alpha_{j}\right) \in G_{n}$. Therefore $D_{n}^{f\left(\alpha_{j}\right)} \subset G_{n}$. Since $n>g\left(f\left(\alpha_{j}\right)\right)$ it follows that $S \cap\left\{\alpha_{m}: m<N_{1}\right\} \subset D_{n}^{f\left(\alpha_{j}\right)} \subset G_{n}$.

Proof of (iii). Obvious.

From $L$ to $\mathbf{N}^{\mathbf{N}}$.

Let $\left\{L_{j}\right\}_{i=1}^{\infty} \in L$. We wish to construct a function which takes $\left\{L_{i}\right\}_{i=1}^{\infty}$ to a sequence $\left\{n_{i}\right\}_{i=1}^{\infty}$ in $\mathbf{N}^{\mathbf{N}}$. To see how this function is to be constructed, suppose $L_{17}=\left(\alpha_{1}, \alpha_{2}, \alpha_{3}, \alpha_{4}, \alpha_{5}\right)$. Here is some information I am going to require:

(i) I will require knowledge of the number 5 (the number of terms in $L_{17}$ ).

(ii) Since this is $L_{17}$, I will require knowledge of the first 17 digits of $\alpha_{1}$, of $\alpha_{2}$, of $\alpha_{3}$, of $\alpha_{4}$, and of $\alpha_{5}$.

(iii) I will require knowledge of whether each term of $L_{17}$ occurred before, when it occurred last, and in what position.

Remark. Maybe $\alpha_{3}$ never occurred before. I require that I know that. Maybe the last time $\alpha_{4}$ occurred was in $L_{10}$, and there it occurred as the twelfth term. I require that I know that.

Above is a collection of information that I require that I must know. Clearly, all this information can be encoded in an integer. We define $n_{17}$ to be the integer which encodes the information I want to know. In this manner we define $\left\{n_{i}\right\}_{i=1}^{\infty}$. We now must establish

(i) If we only alter finitely many of the $L_{i}$, then we only alter finitely many of the $n_{i}$.

(ii) If we alter infinitely many of the $L_{i}$ then we alter infinitely of the $n_{i}$.

Proof of (i). Suppose we only alter $L_{i}$ for some of the $i$ where $i<10$. For each $i$, let $A_{i}=L_{i}$ if $L_{i}$ is not altered, and let $A_{i}$ be the altered $L_{i}$ if $L_{i}$ is altered. For any $\alpha \in \bigcup_{i=1}^{10} L_{i} \cup A_{i}$ define $F(\alpha)$ to be the least $i>10$ where $\alpha \in L_{i}\left(F(\alpha)=10\right.$ if no such $i$ exists). It is easy to see that no value of $n_{i}$ is altered for any $i>\sup \left\{F(\alpha) \mid \alpha \in \bigcup_{i=1}^{10} L_{i}\right\}$.

Proof of (ii). Our procedure will be to merely show that if we alter $L_{17}$ then one of the $n_{i}$ will be altered. The reader will then be able to easily see (ii) for himself.

Suppose $\left\{L_{i}\right\}_{i=1}^{\infty}$ and $\left\{\widehat{L}_{i}\right\}_{i=1}^{\infty} \in L$ are not identical. In particular suppose $L_{17}=\left(\alpha_{1}, \alpha_{2}, \alpha_{3}, \alpha_{4}, \alpha_{5}\right), \hat{L}_{17}=\left(\hat{\alpha}_{1}, \hat{\alpha}_{2}, \hat{\alpha}_{3}, \hat{\alpha}_{4}, \hat{\alpha}_{5}\right)$.

Let us presume that $\alpha_{4}$ and $\hat{\alpha}_{4}$ differ on the 1000th digit. We do not specify whether or not there are any other differences between $\left\{L_{i}\right\}_{i=1}^{\infty}$ and $\left\{\widehat{L}_{i}\right\}_{i=1}^{\infty}$ other than that one digit of $\alpha_{4}$. We claim that the sequences in $\mathbf{N}^{\mathbf{N}}$ that they code to must be different. We use proof by contradiction. We will assume that they both code to the same $\left\{n_{i}\right\}_{i=1}^{\infty} \in \mathbf{N}^{\mathbf{N}}$. 
The proof does not necessarily work (i.e. no contradiction is obtained) if $\alpha_{4}$ only occurs finitely many times in $\left\{L_{i}\right\}_{i=1}^{\infty}$; however since there are only finitely many such values $\alpha$ in $\bigcup_{i=1}^{\infty} L_{i}$ and since we will eventually want to change infinitely many of the $L_{i}$, this possibility need not be considered.

In other words, we can assume $\alpha_{4}$ occurs infinitely many times in $\left\{L_{i}\right\}_{i=1}^{\infty}$. Let us assume that the next time $\alpha_{4}$ occurs, after $L_{17}$, is as the 13 th member of $L_{32}$, which will be denoted by $\alpha_{13}^{32} . \alpha_{13}^{32}=\alpha_{4}$ and the fact that $\alpha_{13}^{32}=\alpha_{4}$ is encoded by $n_{32}$. Since $n_{32}$ is also the 32 nd term coded to by $\left\{\widehat{L}_{i}\right\}_{i=1}^{\infty}$ it follows that the 13 th term of $\widehat{L}_{32}$ (which we denote by $\hat{\alpha}_{13}^{32}$ ) equals $\hat{\alpha}_{4}$. $\hat{\alpha}_{13}^{32}=\hat{\alpha}_{4}$. Now suppose that the next time $\alpha_{4}$ occurs, after $L_{32}$ is as the eighth term of $L_{52}$ which we denote as $\alpha_{8}^{52}$. Now $\alpha_{8}^{52}=\alpha_{4}=\alpha_{13}^{32}$ so by reasoning we just used, the eighth term of $\widehat{L}_{52}, \hat{\alpha}_{8}^{52}$, exists and equals $\hat{\alpha}_{13}^{32}$. Therefore $\hat{\alpha}_{8}^{52}=\hat{\alpha}_{13}^{32}=\hat{\alpha}_{4}$. Continuing this reasoning over and over again we eventually get a number over 1000 (say 1035) and some arbitrary number (say 15) such that if we let $\alpha_{15}^{1035}$ and $\hat{\alpha}_{15}^{1035}$ be the 15 th terms of $L_{1035}$ and $\widehat{L}_{1035}$, respectively, then we can prove $\alpha_{15}^{1035}=\alpha_{4}$ and $\hat{\alpha}_{15}^{1035}=\hat{\alpha}_{4}$. Since both $\left\{L_{i}\right\}_{i=1}^{\infty}$ and $\left\{\widehat{L}_{i}\right\}_{i=1}^{\infty}$ code to the same $n_{1035}$, it follows that $\alpha_{15}^{1035}$ and $\hat{\alpha}_{15}^{1035}$ have the same first 1035 digits. Thus $\alpha_{4}$ and $\hat{\alpha}_{4}$ have the same 1035 digits. This is false. $\alpha_{4}$ and $\hat{\alpha}_{4}$ differ on their 1000th digit.

(e) From $\mathbf{N}^{\mathbf{N}}$ to $\{0,1\}^{\mathbf{N}}$.

Let $\left\{n_{i}\right\}_{i=1}^{\infty} \in \mathbf{N}^{\mathbf{N}}$. For all pair $i, j \in \mathbf{N}$, let

$$
a_{i, j}=\left\{\begin{array}{l}
1 \text { if } \quad j=n_{i} \\
0 \text { else. }
\end{array}\right.
$$

We define $F: \mathbf{N}^{\mathbf{N}} \rightarrow\{0,1\}^{\mathbf{N}}$ by

$$
F\left(\left\{n_{i}\right\}_{i=1}^{\infty}\right)=\left(a_{0,0}, a_{1,0}, a_{0,1}, a_{2,0}, a_{1,1}, a_{0,2}, a_{3,0}, \ldots\right) .
$$

The reader can easily verify himself that $t$ is good.

\section{ACKNOWLEDGMENT}

I would like to thank the referee, who provided one of the proofs (indicated in the text).

Delft University of Technology, Faculty of Technical Mathematics and InformatICS, P. O. Box 356, 2600 AJ Delft, Netherlands 